\title{
Intranasal Ginsenoside Rb1 Targets the Brain and Ameliorates Cerebral Ischemia/Reperfusion Injury in Rats
}

\author{
Tingting Lu, Yongjun Jiang, Zhiming Zhou, Xuanye Yue, Ning Wei, Zhaoyao Chen, Minmin Ma, \\ Gelin XU, and Xinfeng LiU* \\ Department of Neurology, Jinling Hospital, Nanjing University School of Medicine; 305 East Zhongshan Road, Nanjing \\ 210002, China. Received March 31, 2011; accepted April 21, 2011; published online May 20, 2011
}

\begin{abstract}
Ginsenoside Rb1 (GRb1) has been shown to benefit many central nervous system (CNS) disorders, including stroke. However, its bioavailability is low after oral administration due to poor absorption. Intranasal administration has been considered as an effective method for central nervous system drug delivery for its brain-targeting effect. Here, whether intranasal GRb1 could ameliorate cerebral ischemia/reperfusion injury was investigated. First, the concentration of GRb1 in brain tissues and plasma after intranasal and intravenous delivery was calculated using HPLC-MS/MS methods in male Sprague-Dawley rats $(250 \pm 10 \mathrm{~g})$. Intranasal GRb1 was considered brain-targeting if the value of the drug targeting index (DTI) was greater than 1 . Rats were subjected to $1.5 \mathrm{~h}$ middle cerebral artery occlusion (MCAO) and were killed $24 \mathrm{~h}$ after reperfusion. The neuroprotective effects were measured using 2,3,5-triphenyltetrazolium chloride (TTC) staining and Nissl staining. Immunoblotting of LC3 and Beclin 1, crucial autophagy-related proteins, was used to monitor the state of autophagy. With a local bioavailability of $\mathbf{1 0 . 2 8}-\mathbf{3 2 . 4 8} \%$ and DTI of 7.35-23.22 in different brain regions, intranasal GRb1 was determined to be brain-targeting. Less infarct volume and more intact neuronal structure were observed in the GRb1 group. GRb1 also restored the elevation of LC3 and Beclin 1. Our work suggests that intranasal GRb1 exerts brain-targeting effects and that a single dose of intranasal GRb1 immediately after MCAO ameliorates ischemia/reperfusion insult. Autophagy is involved in these beneficial effects.
\end{abstract}

Key words Ginsenoside Rb1; intranasal administration; autophagy; ischemia

Panax ginseng is a highly valued medicinal herb in Eastern society. It has also been well accepted in the West over the past decades. Ginsenosides, a major group of phytoestrogens, are the main active components of ginseng. Ginsenoside $\mathrm{Rb} 1$ (GRb1) is one of the most abundant ginsenosides in ginseng extract, accounting for $27-42 \%$ of the total ginsenosides. ${ }^{1)}$ It has been proven to alleviate many central nervous system (CNS) disorders, including ischemic stroke. The mechanisms of GRb1 in ameliorating cerebral ischemia/reperfusion involve anti-oxidation, ${ }^{2)}$ up-regulating neurotrophic factors, ${ }^{3)}$ anti-apoptosis ${ }^{3,4)}$ and neurogenesis induction. ${ }^{5)}$

However, recent data on the pharmacokinetics of $\mathrm{Rb} 1$ show that its absolute oral bioavailability is as low as $4.35 \%$ in rats. Low membrane permeability dominates the extent of absorption. ${ }^{6,7)}$ Also, some studies have demonstrated that the effects of $0.1-100 \mathrm{nmol} / \mathrm{kg}$ intracerebroventricular GRb1 on ischemic injury are comparable to that of several $\mathrm{mg} / \mathrm{kg}$ GRb1 systemically administrated. ${ }^{3,4,8)}$ These results suggest that GRb1 might have difficulty entering the CNS. The blood-brain barrier (BBB) or other specialized CNS barriers are the main obstacles to the delivery of many potentially therapeutic and diagnostic compounds to specific areas of the brain. Large ( $>500 \mathrm{Da})$ hydrophilic molecules such as peptides and proteins are generally excluded, unless they can be transferred by specific receptor-mediated transcytosis or by the less specific adsorptive-mediated transcytosis. ${ }^{910)}$ With a relatively large molecular weight of 1109.46 and an amphiphilic molecular structure, $\mathrm{Rb} 1$ is predicted too hard to penetrate the BBB.

In recent years, intranasal delivery has gained a significant amount of attention as a convenient and reliable noninvasive method for the systemic administration of drugs. Moreover, it has been proven to rapidly target therapeutics to the CNS, bypassing the $\mathrm{BBB}$, which is particularly suitable for CNS drugs with a large molecular weight and systemic side effects. ${ }^{11)}$ Intranasal Ginsenoside Rg3 has been shown to have anti-fatigue effects. ${ }^{12)}$ The distribution of Panax notoginseng saponins (containing GRb1) in the form of its suspension in plasma after intranasal administration has been tested. ${ }^{13)}$ However, the distribution of ginsenosides in the brain after intranasal or any other delivery method is still unknown.

Macroautophagy (autophagy) is an evolutionarily conserved mechanism for the degradation of cellular proteins and organisms. The process of autophagy is the assembly and transformation of double-membrane vesicles. ${ }^{14)}$ In the last decade, it has been recognized to play a critical role in removing protein aggregates, as well as damaged or excess organelles, to maintain intracellular homeostasis and keep the cell healthy. However, excessive or inappropriate autophagy can also lead to cell death (autophagy related cell death). ${ }^{15}$ ) In the brain, autophagy occurs at a low level under physiological conditions. However, this pathway is rapidly up-regulated under environmental stress ${ }^{16)}$ including focal cerebral ischemia. ${ }^{17)}$ GRb1 has been proven to ameliorate cerebral ischemic insults. ${ }^{3,8)}$ Whether the state of autophagy after focal cerebral ischemia could be influenced by GRb1 has remained unknown.

Hence, the aim of the present study was to investigate the brain-targeting effects of intranasal GRb1 and its influence on cerebral ischemia/reperfusion, with an emphasis on autophagy in rats.

\section{MATERIALS AND METHODS}

Animals Male Sprague-Dawley rats (weight $250 \pm 10 \mathrm{~g}$ ) were provided by the Research Animal Center of Jinling Hospital. Rats were housed with food and water ad libitum. All of the procedures were performed under the National In- 
stitutes of Health (NIH) Guide for the Care and Use of Laboratory Animals (NIH publication No. 80-23, revised 1996).

Drug Administration The intranasal administration procedure was performed as previously described. ${ }^{18)}$ In brief, a rat was placed on its back under anesthesia (chloral hydrate, $400 \mathrm{mg} / \mathrm{kg}$, intraperitoneally (i.p.)). A total volume of $100 \mu \mathrm{l} \mathrm{GRb1}$ (Nanjing Ze-Long Pharmaceutical Co., Ltd., China) or saline was given through both nostrils individually, $10 \mu \mathrm{l}$ at a time, in 3-min intervals. The mouth and the opposite nostril were kept shut during the procedure. For intravenous administration, $100 \mu \mathrm{l}$ GRb1 solution was administrated as a single bolus injection into the tail vein under anesthesia. For intraventricular injection, rats were anesthetized (chloral hydrate, $400 \mathrm{mg} / \mathrm{kg}$, i.p.) and placed in a stereotaxic frame. 3-MA, a selective inhibitor of autophagy, diluted in $10 \mu \mathrm{l}$ saline was injected directly into the lateral cerebral ventricle under the guide of stereotaxis. The following coordinates were used: $0.5 \mathrm{~mm}$ rostral to the bregma, $3.5 \mathrm{~mm}$ lateral to the midline and $5.5 \mathrm{~mm}$ ventral to the skull surface. The body temperature was maintained at $37^{\circ} \mathrm{C}$ by a heat plat.

GRb1 Distribution after Intranasal and Intravenous Administration in Healthy Rats. Sample Collection and Processing Before the experiments, the rats were fasted overnight and divided randomly into intranasal group and intravenous group. GRb1 was administrated intranasally or intravenously at a dose of $12.5 \mathrm{mg} / \mathrm{kg}$ following the method described above. Then, the rats were sacrificed under deep anesthesia at 5, 15, 30, 60, 120 and $180 \mathrm{~min}$ after drug administration. The olfactory lobes, cerebrum, hippocampus, medulla oblongata and cerebellum, as well as plasma were collected. After weighing, the brain tissue samples were homogenized with saline $(1: 5)$. Astragaloside A $(1 \mu \mathrm{g} / \mathrm{ml}$ in methanol, internal standard, the National Institute for the Control of Pharmaceutical and Biological Products, Beijing, China) $50 \mu \mathrm{l}$ was added to $0.5 \mathrm{ml}$ of brain homogenate or plasma. The sample was then vortexed with $2.5 \mathrm{ml}$ acetone-methanol $(4: 1)$ for $3 \mathrm{~min}$ and centrifuged at $1000 \times \boldsymbol{g}$ for $10 \mathrm{~min}$. The supernatant was next collected and evaporated to dryness. The resulting residue was dissolved in $150 \mu \mathrm{l}$ of mobile phase $(0.1 \%$ formic acid : $0.1 \%$ formic acid in methanol $=20: 80$ ) and centrifuged at $15000 \times \boldsymbol{g}$ for $10 \mathrm{~min}$. Following, $20 \mu \mathrm{l}$ of supernatant was injected onto the HPLC column.

HPLC-MS/MS The concentration of GRb1 in plasma and brain tissues was determined by HPLC-MS/MS with a Finnigen TSQ HPLC-MS/MS system (Thermo Fisher Scientific, U.S.A.). Chromatographic separation was achieved on an ODS-2 column $(150 \mathrm{~mm} \times 4.6 \mathrm{~mm}, 5 \mu \mathrm{m}$, Hanbon Hedera) at $30{ }^{\circ} \mathrm{C}$. The mobile phase consisted of $0.1 \%$ formic acid (A) and $0.1 \%$ formic acid in methanol (B). The following program was employed: $0 \mathrm{~min}(80 \% \quad \mathrm{~B}) \rightarrow 0.8 \mathrm{~min}(98 \%$ $\mathrm{B}) \rightarrow 4.5 \mathrm{~min}(98 \% \mathrm{~B}) \rightarrow 4.6 \mathrm{~min}(80 \% \mathrm{~B}) \rightarrow 5.5 \mathrm{~min}(80 \% \mathrm{~B})$ at a flow rate of $1.0 \mathrm{ml} / \mathrm{min}$.

Samples were ionized by positive-ion electrospray ionization (ESI) with an ESI spray voltage of $5 \mathrm{kV}$, atomization air pressure of $35 \mathrm{psi}$, heated capillary temperature of $350^{\circ} \mathrm{C}$, and a collision gas argon pressure of $1.6 \mathrm{mTorr}$. Selected reaction monitoring (SRM) was used for quantification at $\mathrm{m} / \mathrm{z}$ $1131.5 @ 53 \mathrm{eV} \rightarrow m / z 365.1$ for $[\mathrm{MH}]+\mathrm{GRb} 1$ and $m / z$ 807.2@53 eV $\rightarrow m / z 627.4$ for [MH]+ astragaloside A. Peak areas for both chemicals were automatically integrated. Se- lectivity and specificity were determined for the drugs in plasma and brain tissue samples. The linear ranges of $\mathrm{Rb} 1$ were $100-10000 \mathrm{ng} / \mathrm{ml}$ for plasma and $5-25000 \mathrm{ng} / \mathrm{g}$ for brain tissue, respectively. Plasma samples that had Rb1 concentrations above the upper limits were diluted for determination.

The drug concentration vs. time curves for plasma and each brain region were plotted. The area under curves (AUCs) were calculated from 0 to $180 \mathrm{~min}$ using the linear trapezoidal method without extrapolation to infinity. The targeting effect of intranasal GRb1 to different brain regions can be evaluated using the drug targeting index (DTI), which is defined as the ratio between $\left(A U C_{\text {brain }} / A U C_{\text {plasma }}\right)_{\text {i.n. }}$ and $\left(A U C_{\text {brain }} / A U C_{\text {plasma }}\right)_{\text {i.v. }}{ }^{19)}$ Intranasal GRb1 is considered brain-targeting if DTI is greater than 1. The higher the DTI is, the further the degree of intranasal GRb1 targeting to the brain should be.

Middle Cerebral Artery Occlusion (MCAO) After anesthesia (chloral hydrate, $400 \mathrm{mg} / \mathrm{kg}$, i.p.), MCAO was performed following the method described previously. ${ }^{18)}$ In brief, the right common carotid artery (CCA), internal carotid artery (ICA) and external carotid artery (ECA) were exposed under an operating microscope. The ECA was then isolated and ligated. A 4-0 nylon suture was inserted into the ICA through the ECA stump and gently advanced approximately $18 \mathrm{~mm}$ past the carotid bifurcation to occlude the middle cerebral artery (MCA). The suture was withdrawn after an ischemic period of $90 \mathrm{~min}$ to induce reperfusion. During the surgical procedure, the rectal temperature was maintained at $37 \pm 0.5^{\circ} \mathrm{C}$ using a heating plate. After the surgery, rats were randomized into control group, GRb1 group and 3-MA group. Rats with sutures inserted but not advanced were served as the sham group. Right after the onset of $\mathrm{MCAO}$, the GRb1 group received intranasal GRb1 $(1.25 \mathrm{mg} / \mathrm{kg}$ or $12.5 \mathrm{mg} / \mathrm{kg})$. The sham group and control group were given the same volume of intranasal saline. The 3-MA group received intraventricular 3-MA $(150 \mu \mathrm{g} / \mathrm{kg})$. Twenty-four hours after reperfusion, the rats were deeply anesthetized with chloral hydrate and decapitated.

\section{2,3,5-Triphenyltetrazolium Chloride (TTC) Staining} A set of samples from the sham group, control group, GRb1 $1.25 \mathrm{mg} / \mathrm{kg}$ and $12.5 \mathrm{mg} / \mathrm{kg}$ group and 3-MA group were used for the infarct volume evaluation. Brains were rapidly removed and sliced into 2 -mm-thick coronal sections. The slices were stained in TTC (Sigma, Germany) for $30 \mathrm{~min}$ at $37^{\circ} \mathrm{C}$ in the dark. Six serial coronal sections from the frontal pole per animal were then scanned. To eliminate the influence from brain edema, infarct volume was calculated as the volume of the contralateral hemisphere minus the non-infarcted volume of the ipsilateral hemisphere with Image $\mathrm{J}$ (U.S.A.) and presented as the percentage of volume of the contralateral hemisphere.

Nissl Staining For Nissl staining, $20-\mu \mathrm{m}$ frozen sections from the sham group, control group and $12.5 \mathrm{mg} / \mathrm{kg}$ group were stained with $0.2 \%$ crystal violet (Sigma-Aldrich, U.S.A.) for $20 \mathrm{~min}$. After rinsing quickly in distilled water, the sections were differentiated in $95 \%$ ethyl alcohol for $1 \mathrm{~min}$. After dehydrated, the slices were mounted and observed under a light microscope.

Western Blotting The protein levels of LC3 and Beclin 1, two crucial proteins for the process of autophagy, were 
evaluated. Cortical tissues in the border of the infarction from the sham group (relevant position), control group and $12.5 \mathrm{mg} / \mathrm{kg}$ group were dissected and homogenized in radio immunoprecipitation assay (RIPA) buffer. Protein concentrations were determined using a bicinchoninic assay (BCA) kit (Pierce, U.S.A.). Equal amounts of protein were separated by $12 \%$ (for microtubule-associated protein 1A light chain 3, LC3) or $10 \%$ (for Beclin 1) sodium dodecyl sulfate-polyacrylamide gel electrophoresis (SDS-PAGE) (Bio-Rad, CA, U.S.A.), and then they were transferred to $0.22 \mu \mathrm{m}$ polyvinylidene difluoride (PVDF) membranes using a TransBlot semi-dry system (Bio-Rad, CA, U.S.A.). The membranes were blocked in 3\% bovine serum albumin (BSA) in Tris-buffered saline with Tween buffer for $1 \mathrm{~h}$ and then incubated overnight at $4{ }^{\circ} \mathrm{C}$ with the following primary antibodies: anti-LC3 (1 : 500, Cell-signaling, U.S.A.), anti-Beclin 1 ( $1: 500$, Abcam, U.K.) and anti- $\beta$-actin $(1: 500$, Santa Cruz, U.S.A.). Then, the membranes were washed and incubated with horseradish peroxidaseconjugated secondary antibody (goat anti-rabbit immunoglobulin G (IgG), $1: 5000$ or goat anti-mouse IgG, 1:5000, Jingmei Biotech Co., Ltd., China) for $2 \mathrm{~h}$ at room temperature. The blots were developed using a chemiluminescence kit (Pierce, U.S.A.) and were exposed to film. The bands on the film were scanned and analyzed with Image J.

Statistical Analysis Data were presented as mean士 S.E.M. The $F$-test was performed for the two group comparisons of the pharmacokinetic data. When the variance was equal, the data were compared using the independent $t$-test. When the variance was unequal, the data were compared by Welch's test. Multiple group comparisons for the infarct volumes and protein levels were performed by one-way analysis of variance (ANOVA), followed by Tukey's test. $p<0.05$ was considered statistically significant.

\section{RESULTS}

Pharmacokinetics of GRb1 in Plasma The calculated GRb1 concentrations in plasma after intranasal and intravenous delivery are shown in Fig. 1. It was found that the GRb1 levels in plasma following intranasal administration were lower than those following intravenous administration at all time points. The bioavailability $(F)$ in plasma for intranasal GRb1 was approximately $1.40 \%$. However, intranasal GRb1 attained peak concentration at as early as $5 \mathrm{~min}$, indicating quick absorption from the nasal cavity to the blood. Continuous absorption was observed, as indicated by the relatively constant concentration at later time points.

Distribution of GRb1 in the Brain The $C_{\max }$ of GRb1 in all brain regions after intravenous delivery was observed $60 \mathrm{~min}$ after administration, and indicated a relatively slow transport pathway. Among the five brain regions tested, the highest concentration of GRb1 appeared in the hippocampus and cerebrum after intravenous delivery at $60 \mathrm{~min}$. After intranasal administration, the brain GRb1 concentration reached a peak level at $5 \mathrm{~min}$, followed by a decline up to $30 \mathrm{~min}$. The concentrations of GRb1 in the olfactory lobes, medulla oblongata and cerebellum after intranasal delivery were comparable to that after intravenous delivery at $5 \mathrm{~min}$ after administration. These results indicated quick and efficient transport of GRb1 from the nasal cavity to the brain.
The concentration of GRb1 in some brain regions, such as the cerebrum and hippocampus, also increased again at $60 \mathrm{~min}$ after intranasal (Fig. 2). The local bioavailability $(F)$ in the brain regions tested ranged from $10.28-32.48 \%$ (Table 1).

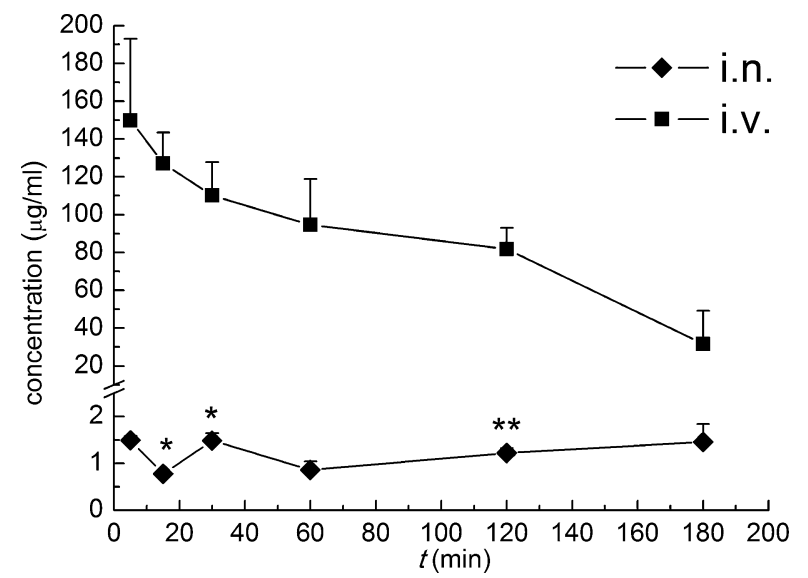

Fig. 1. Pharmacokinetic Profile of Ginsenoside Rb1 $(12.5 \mathrm{mg} / \mathrm{kg})$ in Rat Plasma after Intravenous (i.v.) or Intranasal (i.n.) Administration

Data are shown as mean \pm S.E.M., $n=3$ for each time point in each group. $* p<0.05$, $* * p<0.01 v$ s. i.v., independent sample $t$-test or Welch's test according to the results of the $F$-test.
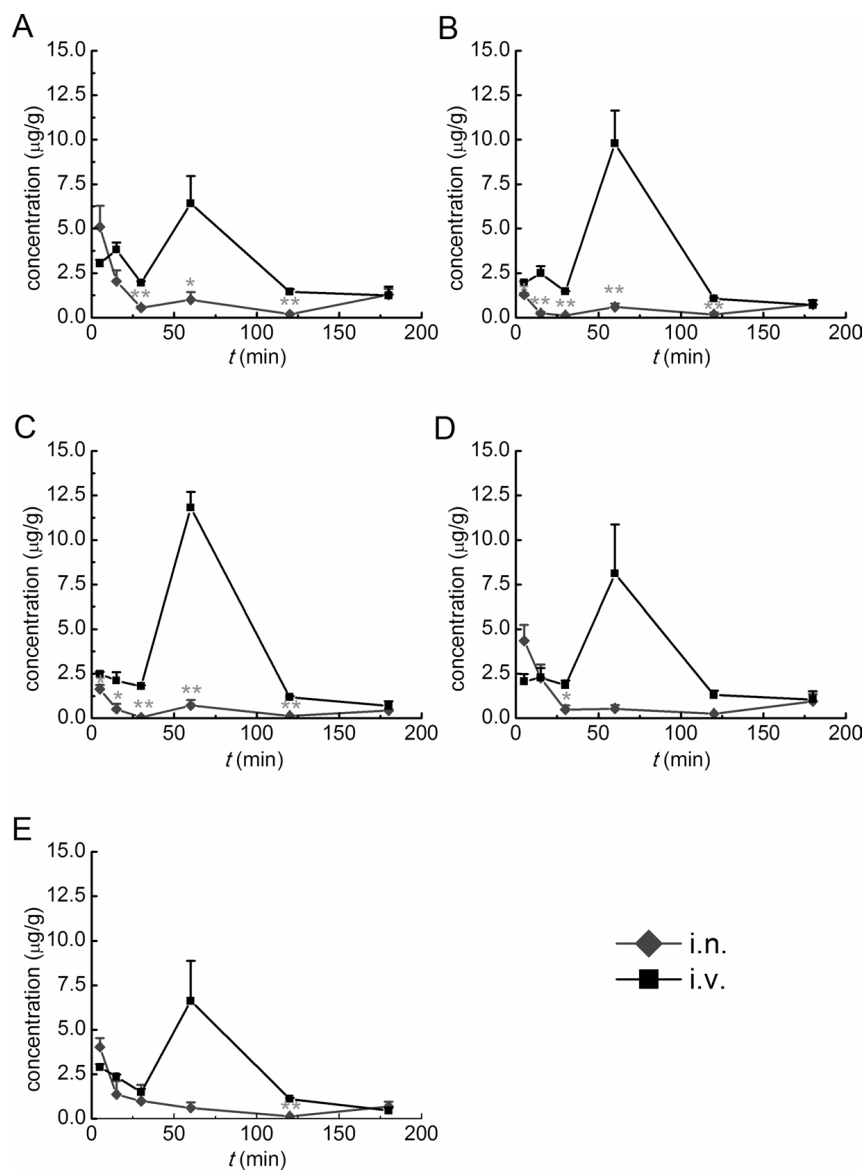

Fig. 2. Pharmacokinetic Profiles of Ginsenoside Rb1 (12.5 mg/kg) in Different Brain Regions after Intranasal (i.n.) or Intravenous (i.v.) Administration in Rats

(A) Olfactory lobes, (B) cerebrum, (C) hippocampus, (D) medulla oblongata and (E) cerebellum. Data are shown as mean \pm S.E.M., $n=3$ for each time point in each group. $* p<0.05, * * p<0.01 v$ s. i.v., independent sample $t$-test. 
Table 1. Local Bioavailability $(F)$ and Drug Targeting Index (DTI) of Intranasal Ginsenoside Rb1

\begin{tabular}{|c|c|c|c|c|c|c|}
\hline Parameters & Plasma & Olfactory lobes & Cerebrum & Hippocampus & Medulla oblongata & Cerebellum \\
\hline$A U C_{\mathrm{i} . \mathrm{V}}\left(\times 10^{3} \mathrm{ng} \cdot \mathrm{min} / \mathrm{g}\right)$ & $15304.91 \pm 3225.93^{a)}$ & $527.75 \pm 101.74$ & $605.01 \pm 100.03$ & $708.69 \pm 60.04$ & $560.93 \pm 167.97$ & $463.30 \pm 116.92$ \\
\hline$A U C_{\mathrm{in}}\left(\times 10^{3} \mathrm{ng} \cdot \mathrm{min} / \mathrm{g}\right)$ & $210.02 \pm 30.60^{a)}$ & $171.44 \pm 48.35$ & $74.55 \pm 24.91$ & $72.89 \pm 25.05$ & $139.64 \pm 44.75$ & $125.64 \pm 56.72$ \\
\hline$F(\%)$ & 1.40 & 32.48 & 12.32 & 10.28 & 24.89 & 27.12 \\
\hline DTI $(\%)$ & - & 23.22 & 8.81 & 7.35 & 17.80 & 19.38 \\
\hline
\end{tabular}

$n=3$ for each time point in each group for $A U C$ calculation. ${ }^{a)} \times 10^{3} \mathrm{ng} \cdot \mathrm{min} / \mathrm{ml}$. The drug targeting index (DTI) is defined as the ratio between $\left(A U C_{\text {brain }} / A U C_{\text {plasma }}\right)_{\text {i.n. }}$ and $\left(A U C_{\text {brain }} / A U C_{\text {plasma }}\right)_{\text {i.v. }}$.
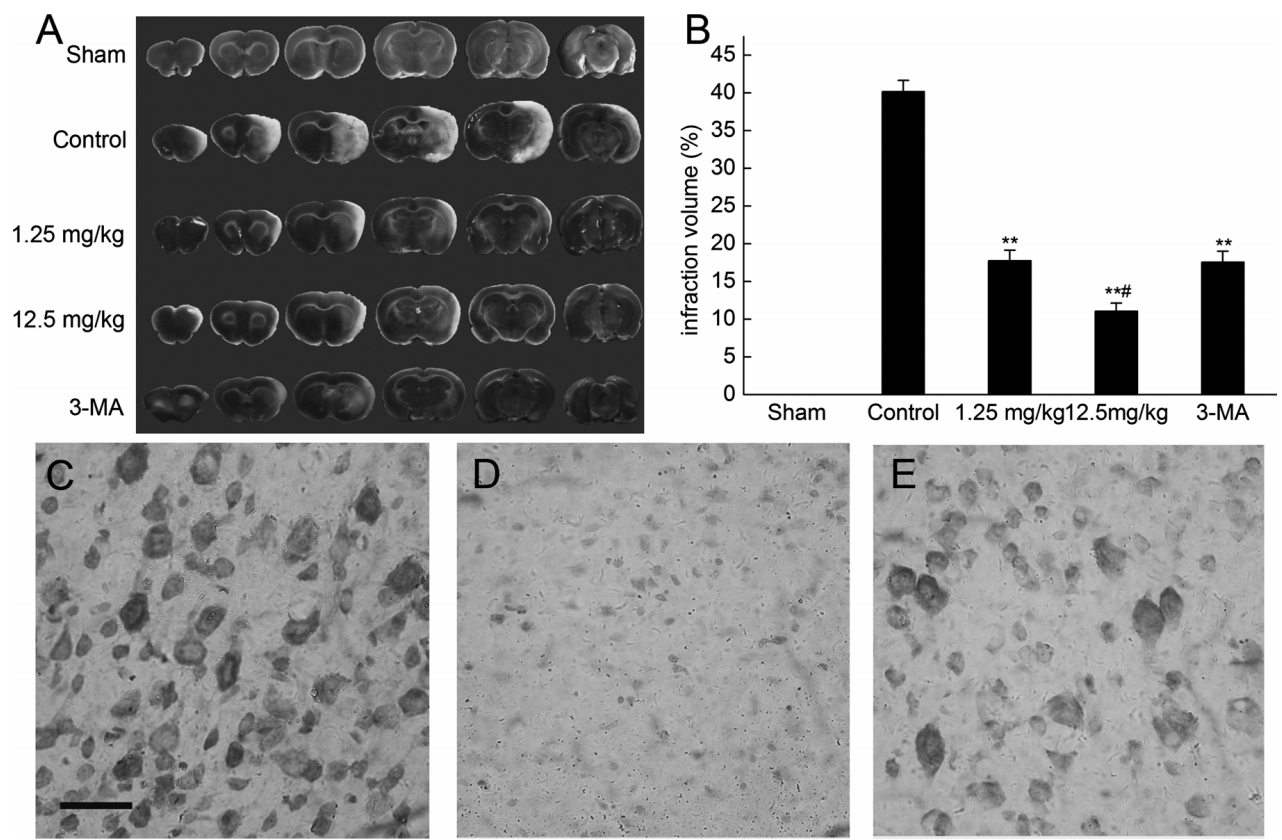

Fig. 3. Neuroprotective Effects of Intranasal Ginsenoside Rb1 after Middle Cerebral Artery Occlusion (MCAO)

Infarct volume is presented as the percentage of the volume of the contralateral hemisphere (A, B). Representative photos of Nissl staining are from the sham group (C), and in the border of the infarction of the control group (D) and $12.5 \mathrm{mg} / \mathrm{kg}$ group (E). Scale bar represents $20 \mu \mathrm{m}$. Data are shown as mean $\pm \mathrm{S}$.E.M., $n=4-5$ in each group. $* * p<0.01 v s$. control group, $\# p<0.05$ vs. $1.25 \mathrm{mg} / \mathrm{kg}$ group, one-way ANOVA, followed by Tukey's test.

Brain-Targeting Effect of Intranasal GRb1 The DTI (Table 1) ranged from 7.35 to 23.22 in different brain regions, which were all greater than 1 . The DTI was most significantly observed in the olfactory lobes, medulla oblongata and cerebellum.

Infarct Volume Twenty-four hours after reperfusion, the infarct volumes were measured by TTC staining. A quantitative assessment revealed that intranasal GRb1 significantly reduced the infarct volume. The infarct volumes were $40.90 \pm 1.66 \%, 17.76 \pm 1.36 \%$ and $11.09 \pm 1.04 \%$ of the ipsilateral hemisphere for the control, $1.25 \mathrm{mg} / \mathrm{kg}$ and 12.5 $\mathrm{mg} / \mathrm{kg}$ GRb1 groups, respectively. A single dose of 3-MA also reduced the infarct volume significantly $(17.59 \pm 1.42 \%)$ (Figs. 3A, B).

Morphological Evaluation under Light Microscopy Nissl staining showed that most neurons in the border of infarction $24 \mathrm{~h}$ after reperfusion were shrunken and only slightly stained in the control group. There were more intact cells in the GRb1 $12.5 \mathrm{mg} / \mathrm{kg}$ group (Figs. 3C-E).

Protein Level of LC3 and Beclin 1 The LC3II and Beclin 1 levels were used to evaluate the autophagy activity. The level of $\beta$-actin served as a loading control. Western blot analysis detected 2 bands at approximately $18 \mathrm{kDa}$ (LC3I) and $16 \mathrm{kDa}$ (LC3II) for LC3. Quantitative analysis revealed that the density of the LC3II band increased after ischemia/reperfusion injury $24 \mathrm{~h}$ after reperfusion $(p<0.01)$. GRb1 treatment suppressed the increasing $(p<0.05)$. Consistent with LC3, the protein level of Beclin 1 was up-regulated after ischemia/reperfusion $(p<0.01)$. Compared to the control group, the Beclin 1 level in the GRb1 group tended to decrease at $24 \mathrm{~h}(p<0.05)$ (Figs. 4A, B).

\section{DISCUSSION}

To the best of our knowledge, this is the first study investigating the distribution of GRb1 in the brain and the influence of intranasal GRb1 on the autophagy state after cerebral ischemia/reperfusion in vivo. Our study showed that intranasal GRb1 exerted significant brain-targeting effects and alleviated ischemia/reperfusion insults in rats; an autophagic mechanism was involved in the benefits.

In recent decades, intranasal administration has gained increasing interest for its convenience, quick absorption and avoidance of first pass elimination. More importantly, drugs delivered intranasally can exert significant brain-targeting effects. In this work, intranasal GRb1 delivery resulted in quick absorption into the brain within $5 \mathrm{~min}$, with comparable drug concentrations to intravenous delivery at this time point. Furthermore, the local bioavailability, which was much higher in the brain regions tested than in the plasma, as well 

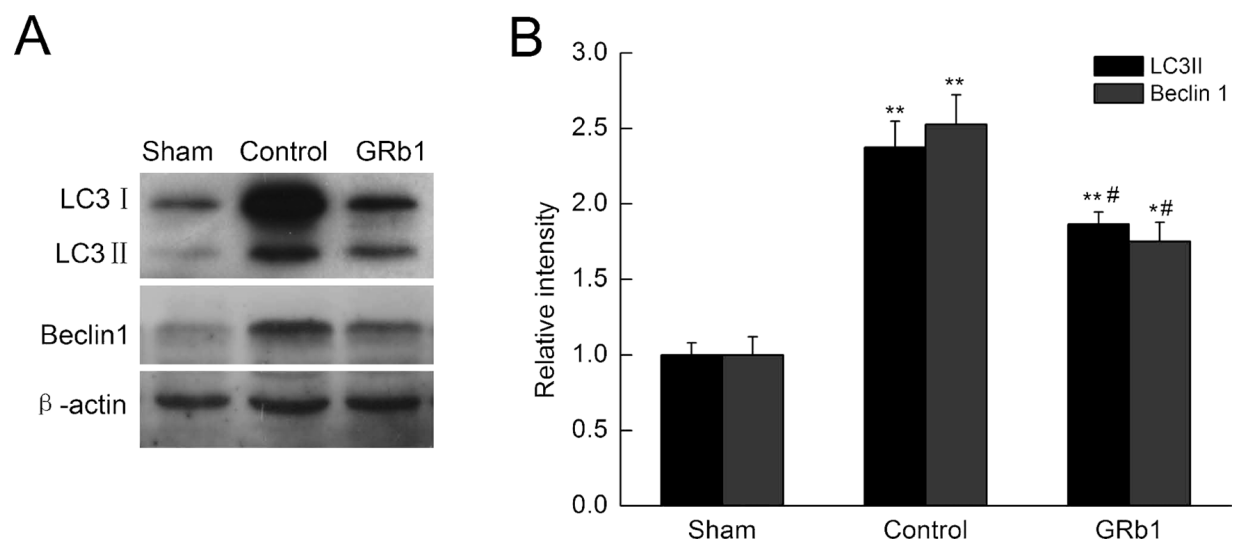

Fig. 4. Modulation of the Protein Level of LC3 and Beclin 1 in the Border of the Infarction $24 \mathrm{~h}$ after Reperfusion by Intranasal Ginsenoside Rb1 $(12.5 \mathrm{mg} / \mathrm{kg})$

(A) Representative bands detected for LC3 and Beclin 1 by immunoblotting. The band for $\beta$-actin served as a loading control. (B) Average density of LC 3 and Beclin 1 protein. Density was determined by using Image J (NIH). Data are shown as mean \pm S.E.M., $n=3$ in each group. $* p<0.05$, $* * p<0.01 v s$. sham group, $\# p<0.05 v s$. control group, one-way ANOVA, followed by Tukey's test.

as the DTI proved intranasal GRb1 to be brain-targeting.

After intranasal administration, drugs can be directly transported from the nasal cavity to the CNS via the olfactory neuroepithelium and trigeminal nerve system. Besides, drugs may also be quickly absorbed into circulation due to the large absorption area and sufficient blood supply of nasal mucosa and then transported to the CNS. ${ }^{11,20)}$ The most significant DTI levels were observed in the olfactory lobes, medulla oblongata and cerebellum, which are close to the encephalic parts of the olfactory epithelia and trigeminal nerve. Therefore, GRb1 is predicted to reach the brain directly through the olfactory epithelia and trigeminal nerve, and is then quickly diffused or distributed into other brain regions. The exact mechanism of drugs transport along nerves has not been completely elucidated. However, drugs with low molecular weights are supposed to be paracellularly absorbed because of their rapid appearances in the CSF and brain. ${ }^{11)}$ Based on its quick presentation in brain regions (within $5 \mathrm{~min}$ ), the nose to CNS transport of GRb1 could occur through this pathway. Besides, our results showed that the GRb1 concentration in the brain rose at $60 \mathrm{~min}$, especially after intravenous administration. These results indicated that GRb1 can be transported from the blood to the brain. For the first time, our results directly proved that GRb1 has the ability to penetrate the $\mathrm{BBB}$, however, in a relatively slow manner.

Though significant brain-targeting effects were observed, the absolute concentration of GRb1 in the brain after intranasal delivery was still much lower than that after intravenous delivery. A concentration advantage would be expected for intravenous administration at the same dose. However, the main aim of this paper was to suggest an easy and comfortable, but ultimately effective, delivery method for GRb1, which is important in emergencies or for long-term administration. Notably, the bioavailability of GRb1 in the brain after intranasal administration $(8.81-23.22 \%)$ was greater than that after oral administration $(4.35 \%){ }^{6}{ }^{6}$ The transportation of GRb1 from the nasal cavity to the brain could be as quick as several minutes after intranasal administration. Moreover, a suitable formulation would greatly improve the bioavailability of intranasal drugs. ${ }^{11,21)}$ Thus, intranasal GRb1 could be a valuable noninvasive delivery method.

As determined by the main direct transport mechanism, the most targeted brain regions for intranasal drugs are the regions next to the nasal cavity. However, it is still encouraging to see brain-targeting effects in other brain regions relatively far from the nasal cavity, such as the hippocampus and hemispheres. For this reason, intranasal administration is acceptable for CNS drug delivery. ${ }^{11,20)}$ In our study, significant brain-targeting effects were also observed in the cerebrum and hippocampus, which are affected by MCAO. Therefore, it is feasible to consider the usage of intranasal GRb1 in cases of ischemia/reperfusion insult.

We proved that intranasal GRb1 could ameliorate ischemia/reperfusion insult in a rat MCAO model. In the range of doses tested, GRb1 reduced the infarct volume, with $12.5 \mathrm{mg} / \mathrm{kg}$ being the most efficient dose. More intact structures of neurons in the GRb1 group were also observed. Intranasal total notoginsenosides (containing GRb1) was shown to reduce the infarct volume in the bilateral common carotid artery occlusion model. ${ }^{13)}$ However, the neuroprotective effects of purified GRb1 after intranasal administration were more intensively observed in our work. We observed that GRb1 could influence autophagy after MCAO in rats.

LC3, a crucial protein for autophagy, is normally located throughout the cytoplasm but becomes concentrated in autophagosomes during autophagy. When associated with autophagosomes, LC3 typically exhibits a different apparent mobility in electrophoresis, changing from $18 \mathrm{kDa}$ (LC3 I) to $16 \mathrm{kDa}$ (LC3 II). LC3 immunoblot analysis is now widely used to monitor autophagy, because the amount of LC3 II clearly correlates with the number of autophagosomes. ${ }^{22} \mathrm{Be}-$ clin 1 was first identified as a B-cell lymphoma-2 (Bcl-2) interacting, coiled-coil protein. It was then proven to be a critical component in the class III PI3 kinase complex (PI3KC3, Vps34) and played a crucial role in the regulation of autophagosomes formation. ${ }^{23}$ Multiple signaling pathways have been proven to regulate autophagy through interaction with Beclin 1.24) The levels of LC3 and Beclin 1 were used to monitor the state of autophagy after MCAO in this work. Consistent with previous works, ${ }^{17,25)} \mathrm{LC} 3$ and Beclin 1 were up-regulated in the borders of the lesion $24 \mathrm{~h}$ after reperfusion, indicating the activation of autophagy. The LC3 and 
Beclin 1 protein levels dropped in the GRb1 group, indicating that GRb1 treatment could restore autophagy $24 \mathrm{~h}$ after reperfusion. This reduction in autophagy-related proteins levels is consistent with the protective effects of GRb1 on ischemia/reperfusion insult, indicating possible involvement of autophagy in the mechanism of GRb1.

The role of elevated autophagy is controversial in ischemic stroke. Some studies conclude that autophagy could be a protective procedure for cells and tissues to sustain hypoxia/ ischemia. ${ }^{2627)}$ On the other hand, there are also a number of papers documenting a deleterious role for autophagy. ${ }^{17,28)} \mathrm{A}$ reliable explanation for these phenomena is that autophagy is primarily a protective process to maintain homeostasis under the stress of cerebral ischemia/reperfusion. However, its excessive or inappropriate activation could also induce cell death. $^{15)}$ 3-MA blocks autophagy through interacting with $\mathrm{PI} 3 \mathrm{KC} 3{ }^{29)}$ In our previous in vitro study, GRb1 and 3-MA both down-regulated autophagy and alleviated glutamateinduced neurotoxicity. ${ }^{30)}$ Consistent with a previous study ${ }^{17}$ ) and our in vitro study, 3-MA successfully alleviated focal ischemia/reperfusion. So a reliable theory is that GRb1 could ameliorate ischemia/reperfusion insults through directly down-regulating autophagy. However, another possible explanation could not be completely excluded, namely that GRb1 can alleviate tissues in the borders of the infarction in the earlier phase of ischemia/reperfusion. Thereby, further activation of autophagy is avoided.

Ginsenosides belong to a family of steroids named steroidal saponins, which share structural features with steroid hormones. Similar to other steroids, ginsenosides are able to intercalate into the plasma membrane and change membrane fluidity and membrane protein structures. This makes ginsenosides able to affect function of multiple membrane proteins, such as ion channels. Moreover, some of the ginsenosides are functional agonists of steroid hormone receptors. ${ }^{31)}$ GRb1 has been proven to activate estrogen receptor (ER)- $\alpha$ and $-\beta{ }^{32)}$ This activation serves as a bias for the effects of GRb1 in some situations, such as attenuating capillary morphogenesis ${ }^{33)}$ and protecting specific neurons against oxidative stress. ${ }^{34)}$ Estrogen can protect the brain from cerebral ischemia through anti-apoptosis, interacting with neurotrophic factors and inducing neurogenesis. ${ }^{35,36)}$ Although the effects of GRb1 are similar to those of estrogen in cerebral ischemia/reperfusion, no direct evidence indicates that GRb1 exerts neuroprotection through activating ER. Interestingly, estrogen is also able to influence autophagy. ${ }^{37,38)}$ Whether GRb1 could modulate autophagy through ERrelated pathways also requires future investigation.

Our results suggest that intranasal GRb1 is an efficient therapy candidate for ischemia/reperfusion. Future studies are warranted to confirm these findings and their clinical applications.

Acknowledgements This study was supported in part by the Nature Science Foundation of China (NSFC 30870848 to GX). The authors thank Genbao Feng for technical advice and assistance.

\section{REFERENCES}

1) Schlag E. M., McIntosh M. S., Phytochemistry, 67, 1510-1519 (2006).

2) Kim Y. C., Kim S. R., Markelonis G. J., Oh T. H., J. Neurosci. Res.,
53, 426-432 (1998).

3) Yuan Q. L., Yang C. X., Xu P., Gao X. Q., Deng L., Chen P., Sun Z. L., Chen Q. Y., Brain Res., 1167, 1-12 (2007).

4) Yoshikawa T., Akiyoshi Y., Susumu T., Tokado H., Fukuzaki K., Nagata R., Samukawa K., Iwao H., Kito G., J. Pharmacol. Sci., 107, 3240 (2008).

5) Gao X. Q., Yang C. X., Chen G. J., Wang G. Y., Chen B., Tan S. K., Liu J., Yuan Q. L., J. Ethnopharmacol., 132, 393-399 (2010).

6) Li X., Wang G., Sun J., Hao H., Xiong Y., Yan B., Zheng Y., Sheng L., Biol. Pharm. Bull., 30, 847-851 (2007).

7) Han M., Sha X., Wu Y., Fang X., Planta Med., 72, 398-404 (2006).

8) Zhang B., Hata R., Zhu P., Sato K., Wen T. C., Yang L., Fujita H., Mitsuda N., Tanaka J., Samukawa K., Maeda N., Sakanaka M., J. Cereb. Blood Flow Metab., 26, 708-721 (2006).

9) Persidsky Y., Ramirez S. H., Haorah J., Kanmogne G. D., J. Neuroimmune Pharmacol., 1, 223-236 (2006).

10) Abbott N. J., Rönnbäck L., Hansson E., Nat. Rev. Neurosci., 7, 41—53 (2006).

11) Dhuria S. V., Hanson L. R., Frey W. H. 2nd, J. Pharm. Sci., 99, 16541673 (2010).

12) Tang W., Zhang Y., Gao J., Ding X., Gao S., Biol. Pharm. Bull., 31, 2024-2027 (2008).

13) Wu Y. J., Zhu X. Y., Sha X. Y., Fang X. L., Yao Xue Xue Bao, 40 $377-381$ (2005).

14) Klionsky D. J., Nat. Rev. Mol. Cell Biol., 8, 931-937 (2007).

15) Mizushima N., Levine B., Cuervo A. M., Klionsky D. J., Nature (London), 451, 1069-1075 (2008)

16) Boland B., Kumar A., Lee S., Platt F. M., Wegiel J., Yu W. H., Nixon R. A., J. Neurosci., 28, 6926-6937 (2008).

17) Wen Y. D., Sheng R., Zhang L. S., Han R., Zhang X., Zhang X. D., Han F., Fukunaga K., Qin Z. H., Autophagy, 4, $762-769$ (2008).

18) Cheng S., Ma M., Ma Y., Wang Z., Xu G., Liu X., Neurol. Res., 31, $753-758$ (2009).

19) Lu W., Jiang W., Chen J., Yin M., Wang Z., Jiang X., Int. J. Pharm., 349, 196-205 (2008).

20) Pires A., Fortuna A., Alves G., Falcão A., J. Pharm. Pharm. Sci., 12, 288-311 (2009)

21) Luppi B., Bigucci F., Cerchiara T., Zecchi V., Expert Opin. Drug Deliv. 7, 811-828 (2010).

22) Klionsky D. J., Cuervo A. M., Seglen P. O., Autophagy, 3, 181-206 (2007).

23) Wang J., Autophagy, 4, 947—948 (2008).

24) Sun Q., Fan W., Zhong Q., Autophagy, 5, 713-716 (2009).

25) Rami A., Langhagen A., Steiger S., Neurobiol. Dis., 29, 132-141 (2008).

26) Ferraro E., Pulicati A., Cencioni M. T., Cozzolino M., Navoni F., di Martino S., Nardacci R., Carrì M. T., Cecconi F., Mol. Biol. Cell, 19, $3576-3588$ (2008)

27) Carloni S., Buonocore G., Balduini W., Neurobiol. Dis., 32, 329-339 (2008).

28) Puyal J., Vaslin A., Mottier V., Clarke P. G., Ann. Neurol., 66, 378389 (2009)

29) Miller S., Tavshanjian B., Oleksy A., Perisic O., Houseman B. T., Shokat K. M., Williams R. L., Science, 327, 1638-1642 (2010).

30) Chen Z., Lu T., Yue X., Wei N., Jiang Y., Chen M., Ni G., Liu X., Xu G., Neurosci. Lett., 482, 264-268 (2010).

31) Lü J. M., Yao Q., Chen C., Curr. Vasc. Pharmacol., 7, 293-302 (2009).

32) Cho J., Park W., Lee S., Ahn W., Lee Y., J. Clin. Endocrinol. Metab., 89, 3510-3515 (2004)

33) Leung K. W., Cheung L. W., Pon Y. L., Wong R. N., Mak N. K., Fan T. P., Au S. C., Tombran-Tink J., Wong A. S., Br. J. Pharmacol., 152, 207-215 (2007).

34) Hwang Y. P., Jeong H. G., Toxicol. Appl. Pharmacol., 242, 18-28 (2010).

35) Lebesgue D., Chevaleyre V., Zukin R. S., Etgen A. M., Steroids, 74, 555-561 (2009).

36) Suzuki S., Brown C. M., Wise P. M., Front. Neuroendocrinol., 30, $201-211(2009)$

37) Sobolewska A., Gajewska M., Zarzynska J., Gajkowska B., Motyl T., Eur. J. Cell Biol., 88, 117-130 (2009).

38) Cosper P. F., Leinwand L. A., Cancer Res., 71, 1710-1720 (2011). 